F todo caso, Picaporte no formuló de ofeo modo un penas entok pero no tardo en destizarse con flexibuidad de xipacmits a las ramas inferiores det to are el suclo

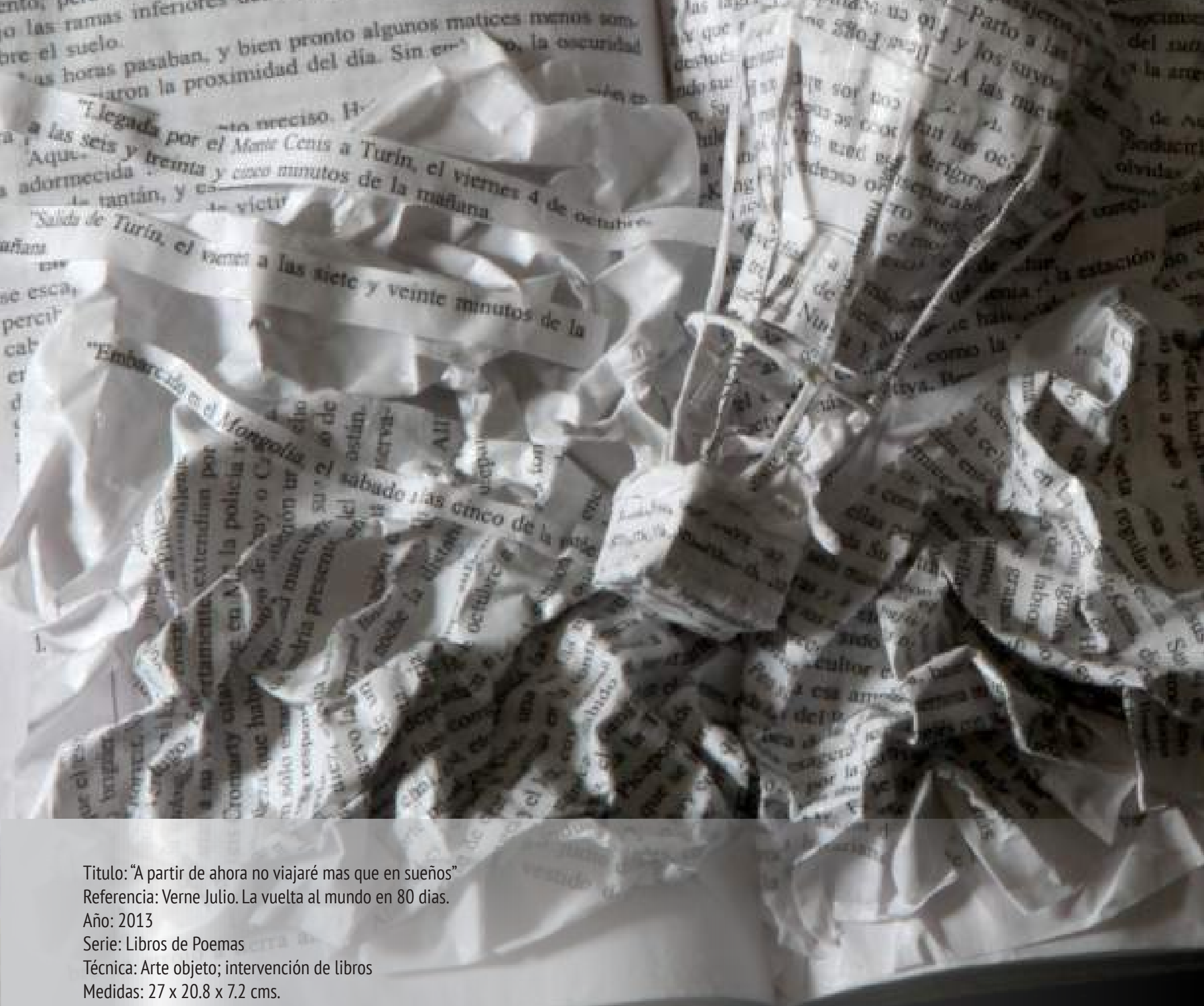

Foto: Pablo Cuéllar 


\title{
Diferencias de género en las actitudes hacia las matemáticas
}

\section{Gender differences in attitudes toward mathematics}

\author{
Martha Patricia de la Torre Gamboa \\ Martín Ramírez Martínez
}

\section{Resumen}

Los resultados de las investigaciones previas no permiten generalizar la existencia de diferencias de género en las actitudes hacia las matemáticas, sin embargo coinciden en su mayoría en señalar que los hombres tienen una mayor motivación extrínseca, que muestran una mayor confianza en sus logros futuros en el área de las matemáticas, menor ansiedad ante las matemáticas y atribución del éxito a causas externas. Las mujeres, por su parte, tienen mejor rendimiento y sobresalientes evaluaciones proporcionadas por los maestros, por otro lado las actitudes de profesores y profesoras, estudiados en las investigaciones. Reflejan claramente una sobrevaloración de lo masculino en detrimento de lo femenino, también coinciden en no encontrar diferencias significativas en la motivación y en el autoconcepto. Pero ¿existen realmente tales diferencias o sólo son producto de estereotipos ya establecidos por los docentes? y, si no existen, ¿por qué tan poca participación femenina en la elección de carreras relacionadas con las ingenierías, la física 0 las matemáticas? Partiendo de estas premisas, el presente estudio trata de atender dos argumentos: Identificar si existen tales diferencias, en particular en alumnos que no se detectan con deficiencias o debilidades aparentes, ni en el rendimiento ni en sus habilidades en matemáticas; y la identificación de diferencias de género en su autoconcepto en el resto de la población estudiantil de primer ingreso al bachillerato.

Palabras clave: género, actitudes, percepción, habilidades, matemáticas, aprendizaje.

\begin{abstract}
The results of previous research do not allow us to generalize the existence of gender differences in attitudes toward mathematics, however they coincide in the majority in pointing out that men have a greater extrinsic motivation, that they show greater confidence in their future achievements in the area of mathematics, less anxiety about mathematics and attribution of success to external causes. Women, on the other hand, have better performance and outstanding evaluations provided by teachers, on the other hand the attitudes of professors and teachers, studied in the investigations. They clearly reflect an overvaluation of the masculine to the detriment of the feminine, they also agree in not finding significant differences in motivation and self-concept. But are there really such differences or are they only the product of stereotypes already established by teachers? and, if they do not exist, why so little female participation in the choice of careers related to engineering, physics or mathematics? Based on these premises, the present study tries to address two arguments: Identify if there are such differences, particularly in students who do not detect themselves with apparent weaknesses, neither in their performance nor in their skills in mathematics; and the identification of gender differences in their self-concept in the rest of the student population of first admission to the baccalaureate.
\end{abstract}

Keywords: gender, attitudes, perception, skills, mathematics, learning. 
C omo todos sabemos la educación media superior en México presenta bajos niveles de logro académico en los resultados de las pruebas estandarizadas, en particular en los resultados de las habilidades numéricas; en los últimos años la investigación académica ha centrado sus esfuerzos en identificar las causas del rechazo que sienten los alumnos por el estudio de esta disciplina, específicamente a finales de los 90 la investigación académica ha cobrado especial interés respecto de las actitudes en el aprendizaje de las matemáticas.

Las relaciones afectivas de los alumnos son parte fundamental en el proceso aprendizaje, esto representa una problemática real en las aulas y constituye una barrera entre la enseñanza del profesor y el aprendizaje del alumno y se traduce en apatía, rechazo, ansiedad, desinterés o miedo por las matemáticas y su correspondiente bajo nivel de desempeño y bajos índices de efectividad académica.

Respecto del comportamiento en el aula, es común entre los profesores de matemáticas notar diferencias entre las actitudes que presentan alumnos y alumnas en el salón de clases.

La interrogante de esta investigación es la siguiente: ¿Existen diferencias entre hombres y mujeres respecto de sus actitudes, autoconcepto y su rendimiento académico en matemáticas a nivel de bachillerato?

La hipótesis se menciona a continuación: existen diferencias determinadas por el género respecto de las actitudes hacia las matemáticas en estudiantes de bachillerato.
El objetivo general es identificar si existen diferencias entre hombres y mujeres respecto de sus actitudes, autoconcepto y su rendimiento académico en matemáticas a nivel de bachillerato.

Y los objetivos específicos son los siguientes:

-Identificar si existen diferencias entre hombre y mujeres respecto de sus actitudes hacia las matemáticas.

- Identificar si existen diferencias entre hombre y mujeres respecto de la forma en que se perciben en sus habilidades en matemáticas. - Identificar si existen diferencias entre hombre y mujeres respecto de su rendimiento en matemáticas.

Existe la necesidad de identificar las causas por las cuales los alumnos presentan rechazo hacia el aprendizaje de las matemáticas, principalmente se han realizado investigaciones para identificar las actitudes de hombres y mujeres respecto del aprendizaje de las matemáticas pero éstas se han realizado en nivel básico y nivel superior y poco, o nada, se ha investigado en el nivel bachillerato.

En los últimos 20 años el estudio de las actitudes y las diferencias de género respecto del aprendizaje de las matemáticas ha tomado fuerza en las investigaciones educativas, los estudios se han realizado en su mayoría en el nivel superior y en el nivel básico principalmente y poco se ha investigado en el nivel medio superior.

Barbero, I., Holgado, F., Vila, E. y Chacón, S. (2007) en su investigación
Existe la necesidad de identificar las causas por las cuales los alumnos presentan rechazo hacia el aprendizaje de las matemáticas". 
titulada Actitudes, hábitos de estudio y rendimiento en Matemáticas: diferencias por género, concluyen en sus resultados que sí que existen diferencias significativas en cuanto al rendimiento en Matemáticas entre los niños y niñas de 13 años en todas las áreas excepto en la correspondiente a los números y operaciones. En relación con los hábitos de estudio o trabajo de las Matemáticas no se encontraron diferencias significativas entre los niños y las niñas pero sí en su actitud hacia las Matemáticas, siendo ésta más favorable en los niños.

Cerezo, T. y Casanova, P. (2011) realizaron una investigación en España y Brasil con alumnos de educación secundaria sobre las Diferencias de género en la motivación académica de los alumnos de Educación Secundaria Obligatoria. Los resultados que obtuvieron reflejan que las chicas presentan menores niveles de motivación extrínseca, se responsabilizan más de sus fracasos, utilizan en mayor medida estrategias de procesamiento de la información y obtienen mejores calificaciones en lenguaje. No se encontraron diferencias de género en autoconcepto académico, en motivación intrínseca, en las atribuciones realizadas ante el éxito y en el rendimiento alcanzado en Matemáticas.

Otras investigaciones (Lightbody et al., 1996; Georgiou, 1999; Powers y Wagner, 1984) han demostrado que existen conductas distintas entre hombres y mujeres; las mujeres tienden a relacionar su rendimiento con el esfuerzo realizado y su fracaso a su falta de habilidades, los hombres lo atribuyen a la habilidad, a la suerte, al profesor y demuestran tener mayor motivación extrínseca respecto de las mujeres.

González, R. M. (2003) realizó un estudio sobre las diferencias de género en el desempeño matemático de estudiantes de secundaria, en sus resultados destaca que el rendimiento en pruebas no presenta diferencias significativas entre hombres y mujeres, respecto de la aptitud, las diferencias promedio son moderadas a favor de los hombres.

En estudios diversos (Mullis et al., 2000; Busselmans-Dehairs et al., 1997; Friedman, 1989) no se han encontrado diferencias significativas entre niños y niñas antes de los trece años. Moore y Smith (1987) realizaron investigaciones con poblaciones específicas donde encontraron grandes diferencias en poblaciones latinas y muy pequeñas diferencias en población negra a favor de los hombres.

Diversas investigaciones sugieren que los patrones de socialización desde la primera infancia suelen favorecer las habilidades visoespaciales y el razonamiento en los niños y limitan a las niñas (Lytton y Romney, 1991), como la construcción de figuras utilizando bloques, armar y desarmar juguetes y rompecabezas, juegos de estrategia y, en general, los deportes que implican movimiento y precisión. En los últimos años estudios de género y matemáticas en México han proporcionado información respecto de que las niñas superan en eficiencia terminal y reprobación escolar en secundaria (Instituto Nacional de Estadística, Geografía e Informática, 2000).

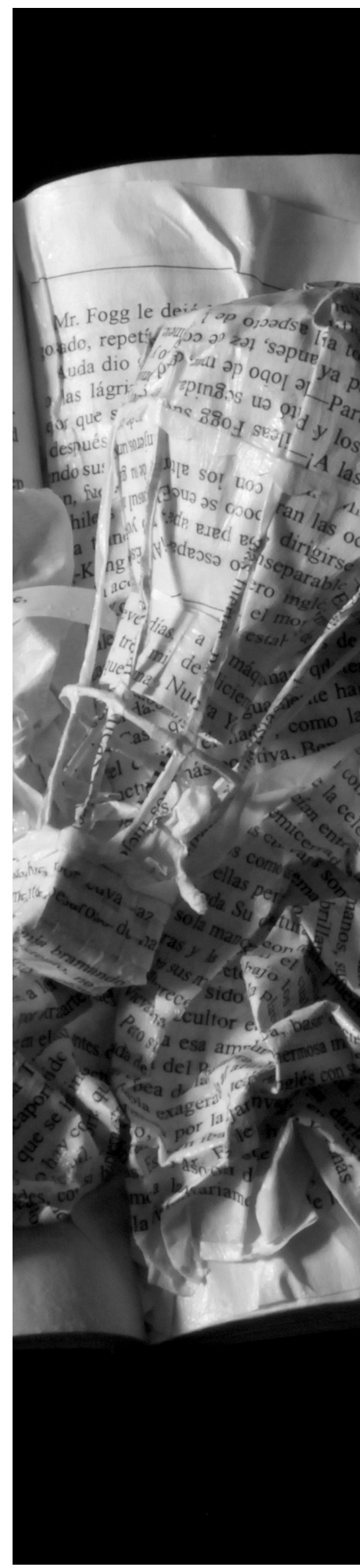

Presencia Universitaria 
Existen también diferencias en las conductas de profesores y profesoras en el aula respecto del género de sus alumnos, Flores, R. (2007), Leder (1987) concluyen respectivamente que el profesorado se dirige más a los hombres que a las mujeres, percibiendo de manera distinta a niños y niñas, y cuando los niños son buenos alumnos lo atribuyen a una mayor creatividad, a la cual consideran indicador de verdadera inteligencia.

Selección de la muestra: Dada la naturaleza de esta investigación se consideró a los alumnos de primer ingreso a preparatoria en agosto 2015 en dos muestras: 106 de los alumnos que obtuvieron al menos el $70 \%$ de aciertos en su examen de ubicación de espacios y 303 alumnos que obtuvieron menos del $70 \%$ de aciertos en su examen de ubicación de espacios.

Instrumento de investigación: Se utilizaron dos instrumentos que fueron aplicados de manera paralela en alumnos de primer ingreso de bachillerato de la Preparatoria 8 de la UANL. Uno de los instrumentos titulado Actitudes matemáticas se aplicó a 106 alumnos que obtuvieron al menos el $70 \%$ de aciertos en el examen de ubicación de espacios en junio del 2015, el otro instrumento fue aplicado a una muestra de 303 alumnos del resto de la población de primer ingreso, este instrumento se nombró Autoconcepto de los alumnos de primer ingreso al bachillerato, ambos instrumentos fueron aplicados en el semestre agosto-diciembre 2015, utilizando escalas decimales. Los datos fueron tratados en el software Statistica 7.0, utilizando la prueba para grupos.
La presente investigación trata de determinar la presencia o no de diferencias de género existentes en distintas variables de actitudes en el aprendizaje de las matemáticas, la investigación tiene un alcance descriptivo con una metodología cuantitativa y un diseño no experimental.

Después de revisar la bibliografía se establecieron los ejes de la investigación y las líneas de trabajo; es importante señalar que no existen estudios específicos sobre la existencia o no de diferencias de género respecto de la autopercepción hacia el aprendizaje de las matemáticas en alumnos que se ha demostrado cuentan con las mismas habilidades matemáticas.

Es sustancial para este estudio retomar las líneas de investigación anteriores y contrastarlas con nuestros resultados para dar respuesta a la hipótesis de la investigación y los objetivos establecidos. Se identifican la población, el tamaño de la muestra y los instrumentos de investigación, se analizan los resultados utilizando la prueba $t$ para grupos, para contrastar la variable género con las variables de los ejes actitudes y percepción de sus habilidades en matemáticas.

Avances de la investigación: La primera muestra la componen 106 alumnos con un nivel de habilidades y conocimientos de matemáticas que van de regulares a muy buenas, las variables utilizadas fueron diseñadas para determinar sus actitudes hacia las matemáticas.

Algunos de los resultados destacados se presentan a continuación:

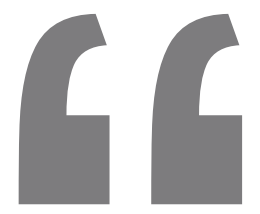

Uno de los

instrumentos

titulado Actitudes

matemáticas

se aplicó a 106

alumnos que

obtuvieron al

menos el $70 \%$

de aciertos en

el examen de

ubicación de

espacios en junio

del 2015". 
La población está compuesta de 53 alumnos y 53 alumnas, se puede inferir que, respecto de la variable de habilidades matemáticas, no se encuentran diferencias significativas.

Para la identificación de posibles diferencias por género se utilizó la prueba estadística $t$ para grupos y la prueba de Levene, apoyados en el software de Statistica, y con un nivel de error menor al 5\%, no se encontraron diferencias significativas para la motivación para e legir más cursos de matemáticas de los estrictamente necesarios; conside- rar que la matemáticas son importantes con su utilidad en los estudios Universitarios.

La flexibilidad en la resolución de problemas; la facilidad o simplicidad al resolver problemas; la facilidad para resolver problemas por diferentes métodos; escuchar a los demás cuando se cometen errores; en la utilización de teoremas, postulados de forma consiente; escribir notas del maestro, escribo las ideas y opiniones interesantes, sentirse motivación en las clases de matemáticas, respecto del rendimiento académico (puntaje de ingreso); en el examen de ubicación de espacios tampoco se identificaron diferencias significativas.

Se identificaron 10 variables para las cuales se encontraron diferencias significativas todas ellas en favor de los hombres (ver la tabla 1). Una vez aplicadas estrategias de actividades en equipos colaborativos y uso de recursos en línea un mes después se aplicó examen de conocimientos matemáticos y se encontraron diferencias significativas en favor de las alumnas (ver tabla 2).

Tabla 1

\begin{tabular}{|c|c|c|c|c|c|c|c|}
\hline & $\begin{array}{c}\text { M } \\
\text { Hombre }\end{array}$ & $\begin{array}{c}\text { M } \\
\text { Mujer }\end{array}$ & $\mathrm{t}$-value & $\mathrm{p}$ & $\begin{array}{l}\text { Std Dev } \\
\text { Hombre }\end{array}$ & $\begin{array}{l}\text { Std Dev } \\
\text { Mujer }\end{array}$ & p Levene \\
\hline $\begin{array}{l}\text { Importancia en la resolución de } \\
\text { problemas cotidianos }\end{array}$ & 8.26 & 7.77 & 2.16 & 0.032 & 1.587 & 1.722 & 0.3388 \\
\hline $\begin{array}{l}\text { Importancia para resolver } \\
\text { problemas en otras disciplinas }\end{array}$ & 8.33 & 7.73 & 2.64 & 0.009 & 1.472 & 1.839 & 0.1980 \\
\hline $\begin{array}{l}\text { El lenguaje matemático es una } \\
\text { herramienta poderosa }\end{array}$ & 7.58 & 6.68 & 3.22 & 0.001 & 1.941 & 2.149 & 0.2866 \\
\hline $\begin{array}{l}\text { Independencia del método de } \\
\text { enseñanza }\end{array}$ & 8.25 & 7.62 & 2.14 & 0.034 & 1.952 & 2.332 & 0.0508 \\
\hline Las matematicas son sencillas & 7.57 & 6.71 & 2.45 & 0.015 & 2.309 & 2.728 & 0.0396 \\
\hline $\begin{array}{l}\text { Altas expectativas de exito en } \\
\text { matemáticas }\end{array}$ & 7.78 & 6.93 & 2.52 & 0.012 & 2.213 & 2.663 & 0.1485 \\
\hline $\begin{array}{l}\text { Puedo aprender con cualquier } \\
\text { maestro }\end{array}$ & 8.19 & 7.35 & 2.69 & 0.008 & 2.035 & 2.534 & 0.0977 \\
\hline $\begin{array}{l}\text { Independencia de los recursos } \\
\text { didácticos del docente }\end{array}$ & 8.27 & 7.48 & 2.94 & 0.004 & 1.738 & 2.157 & 0.0372 \\
\hline Agrado por las matemáticas & 7.69 & 6.90 & 2.07 & 0.039 & 2.435 & 2.992 & 0.1104 \\
\hline $\begin{array}{l}\text { Preferencia de las matemáticas } \\
\text { respecto a otras disciplinas }\end{array}$ & 7.69 & 6.90 & 2.07 & 0.039 & 2.435 & 2.992 & 0.1104 \\
\hline
\end{tabular}

$\mathrm{M}=$ media. $\quad$ Std Dev= Desviación estándar

Tabla 2

\begin{tabular}{|cccccccc}
\hline Resultado de primer parcial & $\begin{array}{c}\mathrm{M} \\
\text { Hombre }\end{array}$ & $\begin{array}{c}\mathrm{M} \\
\text { Mujer }\end{array}$ & t-value & $\mathrm{p}$ & $\begin{array}{c}\text { Std Dev } \\
\text { Hombre }\end{array}$ & $\begin{array}{c}\text { Std Dev } \\
\text { Mujer }\end{array}$ & p Levene \\
\hline & 84.047 & 89.000 & -2.255 & 0.025 & 17.314 & 14.549 & 0.1954 \\
\hline
\end{tabular}


La aplicación del segundo instrumento tenía como objetivo identificar la existencia o no de diferencias de género relacionadas con la percepción de las habilidades, hábitos, conductas en alumnos que obtuvieron menos del $70 \%$ de aciertos en el examen de ubicación de espacios. No se encontraron diferencias significativas por género respecto de las siguientes variables: tiempo dedicado al estudio, percepción del grado de desarrollo de sus habilidades cognitivas, desarrollo de actitudes positivas para el estudio. Tampoco se encontraron diferencias sobre la consideración de que las condiciones del aula afectan su rendimiento escolar ni respecto de la opinión de la necesidad de disciplina en el aula para un mejor rendimiento académico.

En cuestión de la autopercepción en aspectos como creatividad, habilidades comunicativas, responsabilidad, flexibilidad, creatividad, motivación hacia el estudio, respecto de percepción de condiciones de justicia, nor- mas, descalificaciones, obstáculos a la creatividad, respeto y pertenencia, tampoco se encontraron diferencias significativas.

Sin embargo se encontraron diferencias significativas solo en dos variables: La necesidad de reconocimiento de los logros en favor de las alumnas y respecto de la autoestima diferencia en favor de los alumnos (ver la tabla 3).

Tabla 3

\begin{tabular}{lcccccc} 
& $\begin{array}{c}\mathrm{M} \\
\text { Hombre }\end{array}$ & $\begin{array}{c}\mathrm{M} \\
\text { Mujer }\end{array}$ & $\mathrm{p}$ & $\begin{array}{c}\text { Std Dev } \\
\text { Hombre }\end{array}$ & $\begin{array}{c}\text { Std Dev } \\
\text { Mujer }\end{array}$ & p Levene \\
\hline Reconocimiento de los logros & 8.7339 & 9.1006 & 0.0454 & 17.018 & 1.4576 & 0.0337 \\
Autoestima positiva & 9.0081 & 8.3520 & 0.0056 & 14.228 & 2.3354 & 0.0000 \\
\hline
\end{tabular}

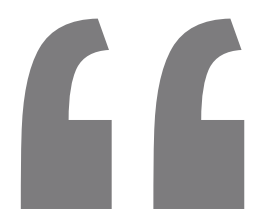

No se encontraron diferencias significativas por género respecto de las siguientes variables: tiempo dedicado al estudio, percepción del grado de desarrollo de sus habilidades cognitivas, desarrollo de actitudes positivas para el estudio". 
La investigación no se ha concluido en su totalidad ya que no se ha realizado el $100 \%$ de los análisis estadísticos previstos para responder. La interrogante de la investigación, probar la hipótesis y atender a los objetivos particulares pero, de manera parcial, se pueden presentar las siguientes conclusiones:

Se acepta la hipótesis de la investigación para la muestra $70 \%$ o más de aciertos en el examen de asignación de espacios, la conclusión con esta población determina que tanto hombres como mujeres tienen el mismo nivel de habilidades. Las mujeres presentan un mejor rendimiento académico en el examen de primer parcial, esto significa que las estrategias de aprendizaje colaborativo per- miten a las mujeres obtener mejores calificaciones, sin embargo sus expectativas de éxito son menores que las de los chicos.

Respecto de los hombres se identificaron diferencias en su favor en aspectos como el nivel de autoestima, ellos conceden a las matemáticas mayor importancia para su vida cotidiana, sus estudios futuros y las prefieren más que a otras disciplinas. Se perciben como más independientes de los métodos de enseñanza y de los profesores y tienen mayores expectativas de éxito académico en matemáticas en relación con las mujeres.

Respecto de la segunda muestra de alumnos, aquellos que no lograron el $70 \%$ de aciertos en el examen de ingreso, prácticamente no se encontraron diferencias significativas en la percepción de sus habilidades, hábitos de estudio, la percepción de la importancia de los ambientes de aprendizaje y autoconcepto.

En esta muestra solo se identificó a las alumnas que necesitan mayor reconocimiento de sus logros académicos por parte de los maestros y que los alumnos tienen una autoestima mayor.

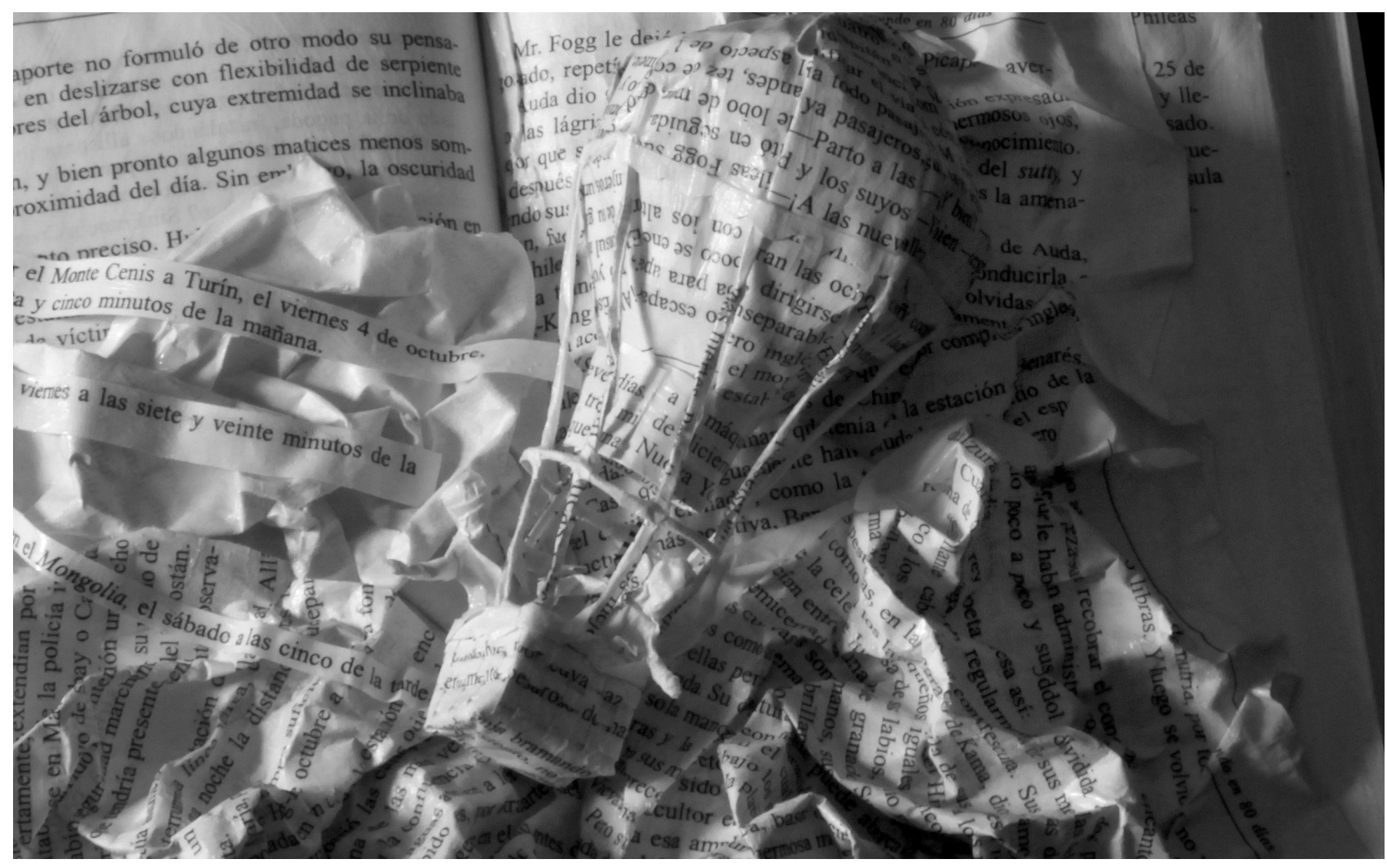



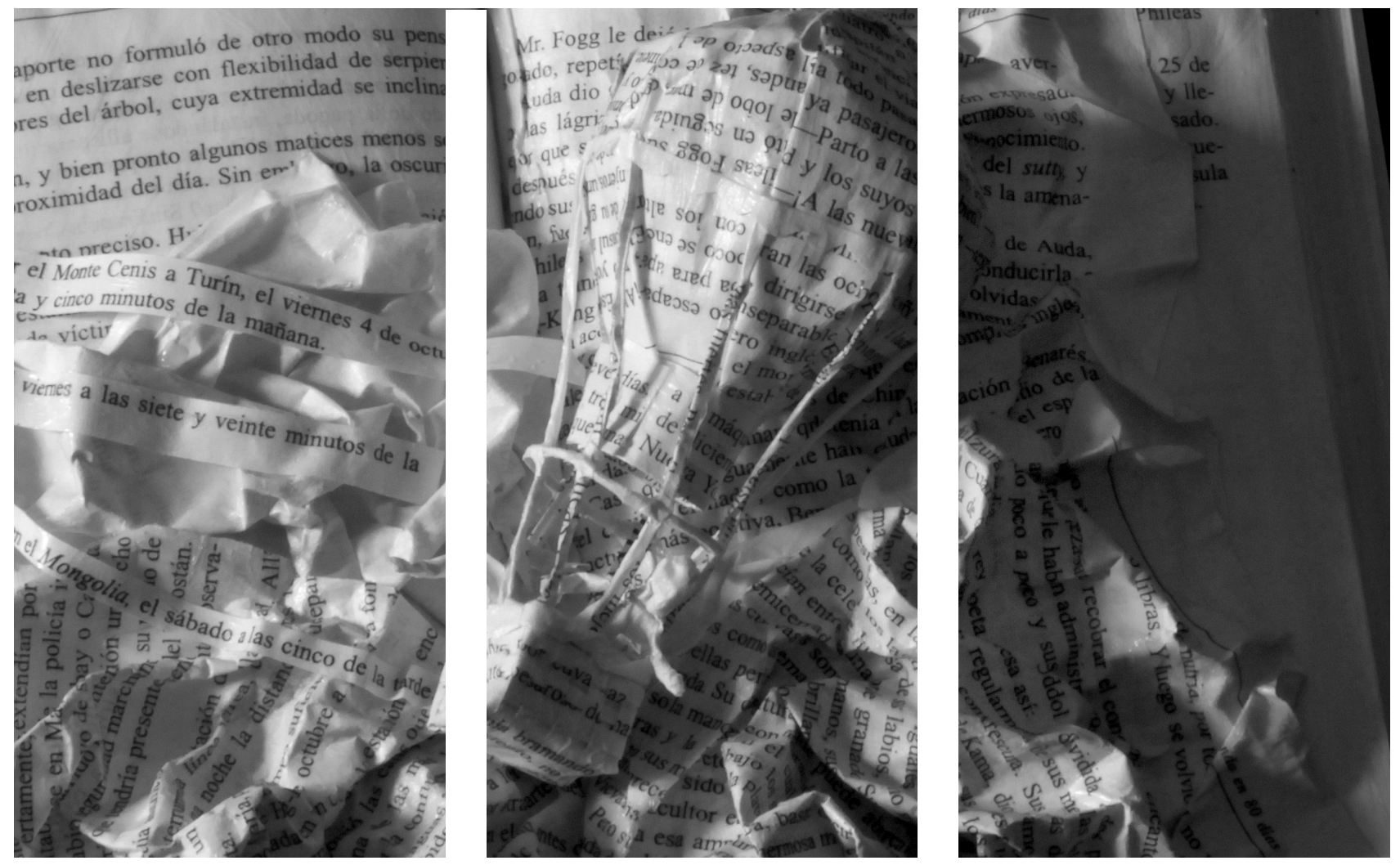

\section{Referencias}

Barbero, I., Holgado, F., Vila, E.y Chacón, S. (2007). Actitudes, hábitos de estudio y rendimiento en Matemáticas: diferencias por género. Red de Revistas Científicas de América Latina, el Caribe, España y Portugal, volumen (19), 413-421.

Cerezo, T., Casanova, P. (2011). Diferencias de género en la motivación académica de los alumnos de Educación Secundaria Obligatoria, Revista electrónica de investigación psicoeducativa, Volumen (2), 97-112.

González, R. M. (2003). Diferencias de género en el desempeño matemático de estudiantes de secundaria. Red de Revistas Científicas de América Latina, el Caribe, España y Portugal, volumen (15), 129-161.

Lightbody, P., Siann, G., Stocks, R. y Walsh, D. (1996). Motivation and attribution at secondary school: the role of gender. Educational Studies, 22,13-25.

Mullis, I. V., M.O. Martin, E.G. Fierros, A.L. Goldberg y S.E. Stemier (2000). Gender Differences in Achievement. IEA's Third International Mathematics and Science Study (TIMSS).

Moore,E.G.yA.W.Smith (1987).Sex and Ethnic Group Differences in Mathematics Achievement: Results from the National Longitudinal Study. Journal for Research in Mathematics Education, núm. 18, pp. 26-36.

Lytton, H. y D.M Romney (1991). Parents Differential Socialization of Boys and Girls: A MetaAnalysis. Psychological Bulletin, vol. 190, núm. 2, pp. 267-296.

Leder, G.C. (1987). La equidad en la clase de matemáticas: ¿realidad o ficción?", equidad y enseñanza de las matemáticas: nuevas tendencias, Madrid. 


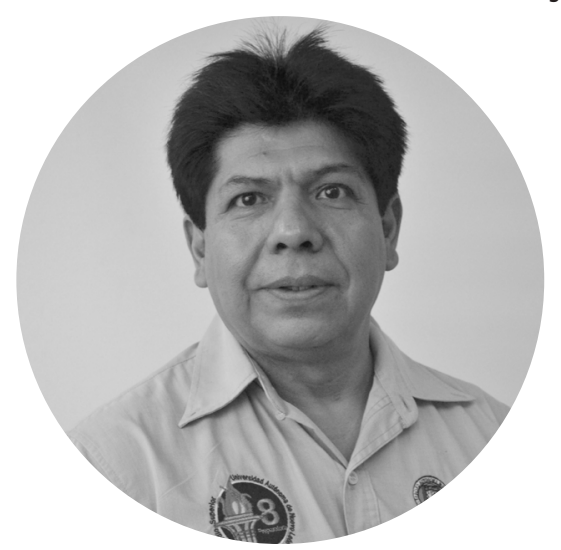

Martín Ramírez Martínez

Ingeniero Agrónomo en Fitotecnia por la Facultad de Agronomía de la UANL, Maestría en Administración de Empresas y Negocios Orientada a Producción y Calidad por la Facultad de Ingeniería Mecánica y Eléctrica de la UANL. Estudios concluidos de Doctorado en Ciencias de la Educación por la Facultad de Ciencia, Educación y Humanidades, de la Universidad Autónoma de Coahuila. Acreditación y Certificación en Competencias Docentes de Nivel Medio Superior por CERTIDENMS. Maestro de tiempo completo en la Escuela Preparatoria 8 de la Universidad Autónoma de Nuevo León.

Correo electrónico: martinramirezmtz@yahoo.com.mx

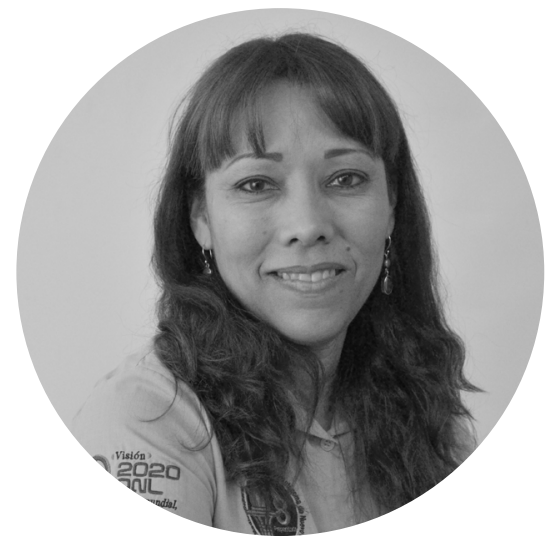

\section{Martha Patricia de la Torre Gamboa}

Licenciada en Ciencias Computacionales por la Facultad de Físico Matemáticas de la UANL, Maestría en Administración con especialidad en Recursos Humanos por la Escuela de Graduados de Ciencias Químicas de la UANL. Estudios concluidos de Doctorado en Ciencias de la Educación por la Facultad de Ciencia, Educación y Humanidades, de la Universidad Autónoma de Coahuila. Acreditación y Certificación en Competencias Docentes de Nivel Medio Superior por CERTIDENMS. Maestra de tiempo completo en la Escuela Preparatoria 8 de la Universidad Autónoma de Nuevo León.

Correo electrónico:

patydelatorrega@gmail.com 